\title{
Genome-wide identification, and phylogenetic and expression profiling analyses, of XTH gene families in Brassica rapa L. and Brassica oleracea L.
}

Di Wu, Anqi Liu, Xiaoyu Qu, Jiayi Liang and Min Song ${ }^{*}$

\begin{abstract}
Background: Xyloglucan endotransglucosylase/hydrolase genes (XTHs) are a multigene family and play key roles in regulating cell wall extensibility in plant growth and development. Brassica rapa and Brassica oleracea contain XTHs, but detailed identification and characterization of the XTH family in these species, and analysis of their tissue expression profiles, have not previously been carried out.

Results: In this study, 53 and 38 XTH genes were identified in B. rapa and B. oleracea respectively, which contained some novel members not observed in previous studies. All XTHs of B. rapa, B. oleracea and Arabidopsis thaliana could be classified into three groups, Group I/II, III and the Early diverging group, based on phylogenetic relationships. Gene structures and motif patterns were similar within each group. All XTHs in this study contained two characteristic conserved domains (Glyco_hydro and XET_C). XTHs are located mainly in the cell wall but some are also located in the cytoplasm. Analyses of the mechanisms of gene family expansion revealed that wholegenome triplication (WGT) events and tandem duplication (TD) may have been the major mechanisms accounting for the expansion of the XTH gene family. Interestingly, TD genes all belonged to Group I/II, suggesting that TD was the main reason for the largest number of genes being in these groups. B. oleracea had lost more of the XTH genes, the conserved domain XET_C and the conserved active-site motif EXDXE compared with B. rapa, consistent with asymmetrical evolution between the two Brassica genomes. A majority of XTH genes exhibited different tissuespecific expression patterns based on RNA-seq data analyses. Moreover, there was differential expression of duplicated XTH genes in the two species, indicating that their functional differentiation occurred after B. rapa and $B$. oleracea diverged from a common ancestor.

Conclusions: We carried out the first systematic analysis of XTH gene families in B. rapa and B. oleracea. The results of this investigation can be used for reference in further studies on the functions of XTH genes and the evolution of this multigene family.
\end{abstract}

Keywords: XTH, Brassica rapa, Brassica oleracea, Tissue expression; comparative genomics

* Correspondence: smin2000@qfnu.edu.cn

Qufu Normal University, College of Life Science, Qufu 273165, P.R. China

C C The Author(s). 2020 Open Access This article is licensed under a Creative Commons Attribution 4.0 International License, which permits use, sharing, adaptation, distribution and reproduction in any medium or format, as long as you give appropriate credit to the original author(s) and the source, provide a link to the Creative Commons licence, and indicate if changes were made. The images or other third party material in this article are included in the article's Creative Commons licence, unless indicated otherwise in a credit line to the material. If material is not included in the article's Creative Commons licence and your intended use is not permitted by statutory regulation or exceeds the permitted use, you will need to obtain permission directly from the copyright holder. To view a copy of this licence, visit http://creativecommons.org/licenses/by/4.0/ The Creative Commons Public Domain Dedication waiver (http://creativecommons.org/publicdomain/zero/1.0/) applies to the data made available in this article, unless otherwise stated in a credit line to the data. 


\section{Background}

The cell wall is an important characteristic structure in plant cells. Cell proliferation and volume increase are inseparable from the process of cell wall reconstruction. Xyloglucan is a component of hemicellulose in the primary cell wall of higher plants. It consists of a cellulose chain with side chains of oligosaccharides, each composed of a few xylose residues. Cell wall reconstruction is accompanied by breakage and regeneration of the cell wall xyloglucan. Xyloglucan endotransglucosylase/hydrolase $(\mathrm{XTH})$ can catalyze the breakage and connection of xyloglucan molecules and modify the fiber-xyloglucan composite structure of plant cell walls, making it one of the key enzymes in cell wall remodeling $[1,2]$.

The XTH family belongs to the glycoside hydrolase family 16 (GH16); common features of proteins in this family are that they adopt a common $\beta$-jelly-roll fold and are active on a range of terrestrial and marine polysaccharides [3-5]. XTH generally performs two catalytic functions, one being xyloglucan endoglucosidase (XEH) activity and the other being xyloglucan endohydrolase (XET) activity, which specifically hydrolyzes xyloglucan glycosidic bonds and promotes cell wall expansion, degradation, repair and morphogenesis [6-8]. Based on the structural characteristics of XTH proteins, they can be divided into three groups, named I/II, III and the early diverging group. Of those reported to date, XTHs with glycosyltransferase activity belong mainly to Group I/II and those with hydrolase activity belong mainly to Group III. There are two conserved domains in XTH proteins, named Glyco_hydro_16 and XET_C. The XET_C domain distinguishes the XTH proteins from other proteins in the GH16 family [8-10].

XTHs are widespread in mosses, lycophytes, ferns, angiosperms and gymnosperms $[9,11-14]$.. Recently, XTH has even been found in algae [12]. XTHs have been widely reported in many plants, including Arabidopsis thaliana (33 genes), Oryza sativa (29 genes), Populus spp. (41 genes), Solanum lycopersicum (25 genes), Nicotiana tabacum (56 genes), Glycine max (61 genes), Hordeum vulgare (24 genes) and Ananas comosus (24 genes) [13-20]. XTH genes show a diversity of tissue expression. In Arabidopsis, AtXTH1, AtXTH21, AtXTH22, AtXTH30 and AtXTH33 are expressed mainly in green siliques, AtXTH24 and AtXTH32 mainly in stems [13]; AtXTH9 is preferentially expressed in flower buds and flower branches, and mutation of this gene resulted in short internodes [21]. XTH proteins are active in the elongation regions of roots and hair cells of vascular plants [22]. Seven $X T H$ genes in rice were found to be specifically expressed in seedling roots [14]. DcXTH2 and DcXTH3 from Dianthus caryophyllus are expressed mainly in petals [23]. XTH activity was detected during fruit expansion in Solanum lycopersicum, Malus domestica, Actinidia chinensis, and strawberry (Fragaria $\times$ ananassa Duch) [24-26]. Constitutive expression of Brassica campestris BcXTH1 caused elongation of flowering branches and increased height in transgenic Arabidopsis plants [27]. Overexpression of cotton (Gossypium spp) GhXTH1 improves cotton fiber length compared with that of wild type plants [28]. XTHs in Ananas comosus are involved in the regulation of fruit ripening and crassulacean acid metabolism and they show tissue specificity [20]. These studies all indicated that XTH is closely linked to plant growth and development.

$X T H$ genes are also associated with plant stress resistance. Overexpression of the Capsicum annuum XTH gene $\mathrm{CaXTH3}$ enhanced drought and salt tolerance, accompanied by an increase in the number of mesophyll cells and changes in leaf shape, in transgenic Arabidopsis and pepper plants $[29,30]$. Overexpression of PeXTH from Populus euphratica in tobacco plants increased their capacity for salt and Cd tolerance [31, 32]. Overexpression of XTH from rose (Rosa rugosa) enhanced drought resistance in transgenic plants [33]. The T-DNA insertion mutants $x$ th 31 , xth 17 and $x$ th15 were more aluminum resistant than the wild type in Arabidopsis [34]. MtXTH3 was induced by $\mathrm{Hg}$ exposure in Medicago truncatula [26]. Some $X T H$ genes are also regulated by hormones, such as gibberellin, brassinosteroids, ethylene and auxin [26].

The Brassicaceae are a large family of plants and many Brassica species are used as oilseed crops, vegetables or feed crops around the world. The Brassica ancestor diverged from a common ancestor with $A$. thaliana approximately 20 million years ago (Mya) followed by a whole genome triplication (WGT) approximately 15.9 Mya. Then the Brassica ancestor diverged to form the modern B. rapa and B. oleracea about 3.75 Mya [3538]. The WGT event brought an increase in genomic materials in Brassica species, making them an excellent model with which to investigate the expansion and evolution of gene families. In addition to genome duplication, tandem duplication (TD) is another important mechanism that induces an increase in the number of members of gene families, i.e. causes gene family expansion [39].

B. rapa and $B$. oleracea are important diploid species in the genus Brassica with completed genome sequencing projects and publicly released genome data, as well as being important as vegetables around the world [40, 41]. Although Behar et al. identified 48 and 27 XTHs in $B$. rapa and $B$. oleracea respectively by mining the JGI Phytozome v12.1 database [9], their characteristics are still unclear. In the present study we identified more XTHs in the genomes of $B$. oleracea and B. rapa using data from different genome versions/resources in three ways. The phylogenetic relationships, gene structures, 
chromosome locations, subgenome distributions, and protein sequences and tissue expression patterns of the XTHs were then analyzed, laying a foundation for further study of XHT gene function in Brassica species and providing useful information for gaining a better understanding of the function and evolution of this gene family in higher plants; the findings may also help researchers to select the most appropriate targets for further genetic engineering and genetic improvement of Brassica crops.

\section{Results}

\section{Identification and characterization of XTHs}

Compared with the 48 and 27 XTHs in $B$. rapa and $B$. oleracea that have previously been reported [9], we identified 53 and 38 XTHs, which include some novel members of the family, while Bol012212 was filtered out in this study because of a lack of the XET_C domain. These genes were designated corresponding to the orthologous XTH genes in Arabidopsis (AtXTH) (Table 1). The identity of BraXTHs and their Arabidopsis orthologs ranged from 61 to $96 \%$, while, the identity of BolXTHs and their Arabidopsis orthologs varying between 57 and 95\% (Additional file 1). Where the final lowercase letter in the gene name is "a", this indicates the highest homology with Arabidopsis, "b" indicates the next highest homology, and so on. The capital letter A or $\mathrm{C}$ in the name indicates, respectively, the $B$. rapa $\mathrm{Ar}$ genome or the $B$. oleracea Co genome. The comparison results of BraXTHs reported in this paper and BraXTHs by Behar et al. [9] are shown in Additional file 2.

No orthologs of AtXTH1, AtXTH2, AtXTH6, AtXTH10, AtXTH14, AtXTH18 or AtXTH19 were found in the $B$. oleracea genome, while the genome of $B$. rapa lacked orthologs of AtXTH1, AtXTH3, AtXTH19 and AtXTH20. Thus more $X T H$ genes have been lost from $B$. oleracea than from $B$ rapa.

The lengths of BraXTHs ranged from 212 (BraA.XTH24.c) to 473 (BraA.XTH3) amino acids, with the molecular weights varying between $24.37 \mathrm{kDa}$ to $55.10 \mathrm{kDa}$, while, the length of BolXTHs ranged from 163 (BolC.XTH29.b) to 346 (BolC.XTH27.a) amino acids, with the molecular weights varying between 18.67 $\mathrm{kDa}$ and $39.87 \mathrm{kDa}$. BraXTH3 was the largest XTH protein in this study. It possesses an ER lumen protein retaining receptor (ER_lumen_recept: InterPro IPR000133, Pfam PF00810) domain in the N-terminal compared with other identified XTHs.

The theoretical PI values for XTHs ranged from 5.06 to 9.58 in B. rapa and $4.96-9.75$ in B. oleracea due to the differences in the polarities of the amino acids making up these proteins. The numbers of introns in $X T H$ genes were relatively similar in the two species; $86.8 \%$ of BraXTH genes and $89.5 \%$ of BolXTH genes had 2-3 introns, of which 24 BraXTHs and 19 BolXTHs had 3 introns, and 22 BraXTHs and 15 BolXTHs had 2 introns. The number of introns in BraA.XTH3 was the largest (7), while BolC.XTH29.b lacked introns.

The Plant-mPLoc server (http://www.csbio.sjtu.edu.cn/ bioinf/plant-multi/) was used to predict the subcellular location of BraXTH and BolXTH proteins. The result showed that all XTH proteins were located on the cell wall. In addition to the cell wall, 20 BraXTHs and 12 BolXTHs were also predicted to localize in the cytoplasm. BraA.XTH3 was found to be located in both the cell wall and the endoplasmic reticulum. XTH localize just were bioinformatic speculation and the real situation will be experimental evidence. The signal peptide prediction results indicated that 46 BraXTHs and 33 BolXTHs had signal peptides.

\section{Phylogenetic analysis of XTH proteins}

In order to investigate the evolutionary relationship among different $X T H$ gene family members, we used the full-length XTH protein sequences from B. rapa, B. oleracea and $A$. thaliana to generate a phylogenetic tree based on the Maximum Likelihood method, using a structurally characterized bacterial lichenase (1GBG, EC 3.2.1.73) as an outgroup (Fig. 1, Additional file 3). Three groups (Early diverging group, Group I/II and Group III) were identified based on clade support values, the topology of the phylogenetic tree, and the previous classification of XTH families in Arabidopsis [6, 13]. So far, $\mathrm{XEH}$ activity has only been reported in clade IIIA [9, 42]. The early diverging close to the root was the smallest group, containing 12 members. There were 11 XTHs in Group IIIA and 20 in Group IIIB. The rest of the XTHs belonged to Group I/II, which included 22 AtXTHs, 35 BraXTHs and 23 BolXTHs. As Fig. 1 shows, XTHs from $B$. rapa and $B$. oleracea were clustered with their $A$. thaliana homologs. There were 41 sister pairs at the termini of phylogenetic tree branches that showed close relationships and 30 of these were orthologous pairs between the $B$. rapa genome and the $B$. oleracea genome.

\section{Structure of XTH genes, pattern of motifs and structure- based sequence alignment in XTH proteins}

Previous studies showed that the exon organization in Arabidopsis XTH genes is well conserved within each group $[13,43]$. To better characterize the structural conservation and diversification of XTH genes during their evolution, the exon-intron organization of the coding sequences of individual $X T H$ genes coding sequence was obtained for members of each group. Each XTH protein in the two species had a Glyco_hydro_16 domain and an XET_C domain. As shown in Fig. 2b, c and Fig. 3b, c, the Glyco_hydro_16 domain spanned the sequence of 
Table 1 Characteristics of XTHs identified in B. rapa and B. oleracea

\begin{tabular}{|c|c|c|c|c|c|c|}
\hline Symbol & BRAD ID & Peptide length (aa) & PI & MW (kDa) & SignalP & Subcellular localization \\
\hline BraA.XTH2.a & Bra001434 & 288 & 9.1 & 32.68 & $\mathrm{~S}$ & Cell wall \\
\hline BraA.XTH2.b & Bra001433 & 284 & 8.5 & 31.94 & S & Cell wall \\
\hline BraA.XTH3 & Bra015093 & 473 & 8.72 & 55.10 & S & Cell wall Endoplasmic reticulum \\
\hline BraA.XTH4 & Bra013164 & 295 & 8.91 & 34.06 & S & Cell wall Cytoplasm. \\
\hline BraA.XTH5.a & Bra006220 & 293 & 9.21 & 34.09 & S & Cell wall Cytoplasm. \\
\hline BraA.XTH5.b & Bra008796 & 279 & 8.86 & 32.48 & - & Cell wall Cytoplasm. \\
\hline BraA.XTH6 & Bra024415 & 285 & 6.06 & 32.75 & S & Cell wall \\
\hline BraA.XTH7 & Bra017855 & 292 & 7.59 & 33.41 & S & Cell wall \\
\hline BraA.XTH8 & Bra019846 & 299 & 5.43 & 35.03 & S & Cell wall \\
\hline BraA.XTH9.a & Bra000840 & 290 & 5.15 & 33.12 & S & Cell wall \\
\hline BraA.XTH9.b & Bra034193 & 344 & 5.06 & 38.88 & _ & Cell wall \\
\hline BraA.XTH1O & Bra040716 & 297 & 8.21 & 34.49 & S & Cell wall \\
\hline BraA.XTH11.a & Bra019552 & 301 & 8.66 & 35.11 & S & Cell wall \\
\hline BraA.XTH11.b & Bra029907 & 278 & 8.7 & 32.59 & S & Cell wall \\
\hline BraA.XTH12.a & Bra002723 & 286 & 5.4 & 32.42 & S & Cell wall Cytoplasm. \\
\hline BraA.XTH12.b & Bra020436 & 283 & 5.11 & 32.24 & S & Cell wall Cytoplasm. \\
\hline BraA.XTH12.c & Bra006814 & 319 & 5.43 & 36.29 & S & Cell wall Cytoplasm. \\
\hline BraA.XTH14.a & Bra019141 & 286 & 6.95 & 32.69 & S & Cell wall Cytoplasm. \\
\hline BraA.XTH14.b & Bra013923 & 284 & 5.96 & 32.20 & S & Cell wall Cytoplasm. \\
\hline BraA.XTH15 & Bra038442 & 289 & 9.41 & 33.03 & s & Cell wall Cytoplasm. \\
\hline BraA.XTH16 & Bra014975 & 291 & 9.14 & 33.20 & S & Cell wall Cytoplasm. \\
\hline BraA.XTH17.a & Bra011181 & 282 & 8.56 & 32.01 & S & Cell wall \\
\hline BraA.XTH17.b & Bra011180 & 282 & 9 & 32.10 & S & Cell wall \\
\hline BraA.XTH17.C & Bra010290 & 280 & 9.3 & 32.03 & S & Cell wall \\
\hline BraA.XTH17.d & Bra024087 & 284 & 8.84 & 32.36 & S & Cell wall \\
\hline BraA.XTH17.e & Bra010291 & 282 & 9.1 & 32.23 & S & Cell wall \\
\hline BraA.XTH18 & Bra024088 & 282 & 9.09 & 32.25 & S & Cell wall \\
\hline BraA.XTH21 & Bra038819 & 239 & 9.12 & 27.27 & _- & Cell wall \\
\hline BraA.XTH22.a & Bra002719 & 283 & 5.96 & 31.98 & S & Cell wall Cytoplasm. \\
\hline BraA.XTH22.b & Bra020433 & 260 & 5.49 & 29.61 & S & Cell wall \\
\hline BraA.XTH22.C & Bra002718 & 214 & 6.72 & 24.39 & - & Cell wall Cytoplasm. \\
\hline BraA.XTH22.d & Bra002720 & 214 & 6.72 & 24.39 & _ & Cell wall Cytoplasm. \\
\hline BraA.XTH22.e & Bra020432 & 283 & 6.33 & 31.79 & s & Cell wall \\
\hline BraA.XTH23.a & Bra008806 & 287 & 5.29 & 32.11 & s & Cell wall Cytoplasm. \\
\hline BraA.XTH23.b & Bra013922 & 287 & 5.29 & 32.11 & S & Cell wall Cytoplasm. \\
\hline BraA.XTH24.a & Bra011179 & 282 & 8.76 & 31.84 & S & Cell wall Cytoplasm. \\
\hline BraA.XTH24.b & Bra024089 & 279 & 8.93 & 31.59 & S & Cell wall Cytoplasm. \\
\hline BraA.XTH24.C & Bra010292 & 212 & 8.76 & 24.37 & - & Cell wall Cytoplasm. \\
\hline BraA.XTH25.a & Bra002722 & 284 & 9.06 & 32.58 & S & Cell wall Cytoplasm. \\
\hline BraA.XTH25.b & Bra020434 & 285 & 7.66 & 32.49 & S & Cell wall Cytoplasm. \\
\hline BraA.XTH26 & Bra011066 & 292 & 9.51 & 33.07 & S & Cell wall \\
\hline BraA.XTH27.a & Bra026630 & 333 & 7.67 & 38.29 & S & Cell wall \\
\hline BraA.XTH27.b & Bra024848 & 323 & 7.73 & 36.95 & - & Cell wall \\
\hline BraA.XTH28 & Bra026809 & 328 & 7.24 & 37.79 & S & Cell wall \\
\hline
\end{tabular}


Table 1 Characteristics of XTHs identified in B. rapa and B. oleracea (Continued)

\begin{tabular}{|c|c|c|c|c|c|c|}
\hline Symbol & BRAD ID & Peptide length (aa) & PI & MW (kDa) & SignalP & Subcellular localization \\
\hline BraA.XTH29.a & Bra012559 & 347 & 9.1 & 40.07 & S & Cell wall \\
\hline BraA.XTH29.b & Bra013367 & 351 & 8.68 & 40.49 & S & Cell wall \\
\hline BraA.XTH3O & Bra035513 & 342 & 8.14 & 39.74 & S & Cell wall \\
\hline BraA.XTH31.a & Bra019416 & 292 & 7.04 & 33.11 & S & Cell wall \\
\hline BraA.XTH31.b & Bra037614 & 292 & 8.21 & 33.57 & S & Cell wall \\
\hline BraA.XTH32.a & Bra005238 & 289 & 9.58 & 34.30 & S & Cell wall \\
\hline BraA.XTH32.b & Bra017220 & 299 & 9.53 & 34.15 & S & Cell wall \\
\hline BraA.XTH32.C & Bra023083 & 299 & 9.49 & 34.25 & S & Cell wall \\
\hline BraA.XTH33 & Bra018433 & 317 & 8.97 & 35.38 & S & Cell wall \\
\hline BolC.XTH2 & Bol003021 & 291 & 8.69 & 32.92 & S & Cell wall \\
\hline BolC.XTH4 & Bol042558 & 295 & 8.91 & 34.12 & S & Cell wall Cytoplasm. \\
\hline BolC.XTH5 & Bol043401 & 295 & 8.87 & 34.27 & S & Cell wall Cytoplasm. \\
\hline BolC.XTH7 & Bol018592 & 292 & 7.6 & 33.40 & S & Cell wall \\
\hline BO/C.XTH8 & Bol036640 & 199 & 4.96 & 23.32 & S & Cell wall \\
\hline BolC.XTH9.a & Bol030618 & 290 & 5.15 & 33.09 & S & Cell wall \\
\hline BolC.XTH9.6 & Bol006115 & 290 & 5.02 & 33.04 & S & Cell wall \\
\hline BolC.XTH11.a & Bol013144 & 209 & 9.32 & 24.68 & - & Cell wall \\
\hline Bo/C.XTH11.b & Bol013146 & 225 & 8.21 & 26.08 & S & Cell wall \\
\hline BolC.XTH12 & Bol026029 & 286 & 5.37 & 32.43 & S & Cell wall Cytoplasm. \\
\hline BolC.XTH13 & Bol012213 & 268 & 5.72 & 30.74 & - & Cell wall Cytoplasm. \\
\hline BolC.XTH15 & Bol027057 & 289 & 9.34 & 33.01 & S & Cell wall Cytoplasm. \\
\hline BolC.XTH16 & Bol037310 & 291 & 9.14 & 33.18 & S & Cell wall Cytoplasm. \\
\hline BolC.XTH17.a & Bol020988 & 282 & 9.01 & 32.12 & S & Cell wall \\
\hline BolC.XTH17.b & Bol033655 & 248 & 8.74 & 28.50 & S & Cell wall \\
\hline BolC.XTH2O & Bol012994 & 187 & 9.56 & 21.28 & S & Cell wall \\
\hline BolC.XTH21 & Bol041548 & 340 & 8.91 & 38.61 & - & Cell wall \\
\hline BolC.XTH22.a & Bol014220 & 283 & 5.8 & 32.07 & S & Cell wall Cytoplasm. \\
\hline BolC.XTH22.b & Bol014219 & 283 & 5.98 & 31.76 & S & Cell wall \\
\hline BolC.XTH23 & Bol039563 & 287 & 5.05 & 32.12 & S & Cell wall Cytoplasm. \\
\hline BolC.XTH24.a & Bol033653 & 279 & 8.77 & 31.59 & S & Cell wall Cytoplasm. \\
\hline BolC.XTH24.b & Bol012996 & 323 & 8.95 & 36.96 & S & Cell wall Cytoplasm. \\
\hline Bo/C.XTH24.C & Bol033652 & 284 & 5.07 & 32.35 & S & Cell wall \\
\hline Bo/C.XTH24.d & Bol020987 & 212 & 8.6 & 24.38 & - & Cell wall Cytoplasm. \\
\hline BolC.XTH25 & Bol014221 & 285 & 7.67 & 32.39 & S & Cell wall Cytoplasm. \\
\hline BolC.XTH26 & Bol019625 & 292 & 9.5 & 33.08 & S & Cell wall \\
\hline BolC.XTH27.a & Bol004698 & 346 & 8.2 & 39.87 & S & Cell wall \\
\hline BolC.XTH27.b & Bol002411 & 269 & 9.12 & 30.57 & S & Cell wall \\
\hline BolC.XTH28 & Bol031516 & 318 & 7.22 & 36.36 & S & Cell wall \\
\hline BolC.XTH29.a & Bol009357 & 271 & 8.64 & 31.02 & S & Cell wall \\
\hline BolC.XTH29.b & Bol024395 & 163 & 9.3 & 18.67 & - & Cell wall \\
\hline Bo/C.XTH30.a & Bol001946 & 343 & 6.55 & 39.68 & S & Cell wall \\
\hline Bo/C.XTH30.b & Bol014054 & 342 & 7.62 & 39.70 & S & Cell wall \\
\hline BolC.XTH31 & Bol041311 & 292 & 7.65 & 33.21 & S & Cell wall \\
\hline Bo/C.XTH32.a & Bol037699 & 299 & 9.57 & 34.11 & S & Cell wall \\
\hline
\end{tabular}


Table 1 Characteristics of XTHs identified in B. rapa and B. oleracea (Continued)

\begin{tabular}{lllllll}
\hline Symbol & BRAD ID & Peptide length (aa) & PI & MW (kDa) & SignalP & Subcellular localization \\
\hline BolC.XTH32.b & Bol039723 & 243 & 9.75 & 27.84 & $\mathrm{~S}$ & Cell wall \\
Bo/C.XTH32.C & Bol001671 & 234 & 9.82 & 26.78 & $\mathrm{~S}$ & Cell wall \\
Bo/C.XTH33 & Bol022041 & 317 & 8.82 & 35.50 & $\mathrm{~S}$ & Cell wall
\end{tabular}

New homologs identified in this study in B. oleracea are shown in bold. The rest BolXTHs have been identified by Behar et al. [9]

motifs 6-4-3-1-2-8, though some proteins lacked one or more of these motifs. The lengths of 4 BraXTHs and 9 BolXTHs, including 7 newly identified XTHs, are less than 250 amino acids, due to the deletion of 1 to 4 motifs from the Glyco_hydro_16 domain (Figs. 2, 3). The XET_C domain mainly covered motifs 5 and 9. Fifteen BraXTHs and 10 BolXTHs also shared motif 10, forming the block 10-5-9. Six BraXTHs and 7 BolXTHs replaced motif 9 with motif 7 , forming a new tandem motif pattern (motif 5-7 in tandem). Overall, motifs had a similar distribution within the same group.

In addition to $X T H 26$, all genes in Group I contained 1-2 introns. Apart from XTH8, all genes in Group II contained 3 introns. All Group III genes in the two species had 3 introns with the exceptions of BolC.XTH29.a and BolC.XTH29.b. Generally, the motif patterns in different XTH proteins showed only small differences, and the genes that clustered in the same group showed similar patterns of gene structure.

The alignments of the XTHs together with PttXET16A (PDB id: 1UN1), a xyloglucan endotransglycosylase with known protein structure [44, 45], were used to predict the secondary structures of the BraXTH proteins and BolXTH proteins using ESPript (http://espript.ibcp.fr/ ESPript/ESPript/) (Additional files 4 and 5). The position of the N-glycosylation site of PttXET16A and BobXET16A with known protein structure, was conserved [44-46]. The site also was conserved in 46 BraXTHs and 28 BolXTHs, but it was not found in 7 BraXTHs and 10 BolXTHs: BolC.XTH31, BolC.XTH32.a, BolC.XTH33, the 7 BolXTHs that lacked the EXDXE conserved active-site motif (BolC.XTH8, BolC.XTH20, BolC.XTH27.b, BolC.XTH29alb and BolC.XTH32.b\c), BraA.XTH2.a, BraA.XTH31.alb, BraA.XTH32.alb $\backslash c$ and BraA.XTH33 (Additional files 4 and 5). Alterations of amino acid residues were found within this catalytic region in AtXTH11 and its homologs. In AtXTH11, EXDXE was replaced by ELCFQ, while it was replaced by GLCFQ in BraA.XTH11b and BolC.XTH11.alb, and by QLCFQ in BraA.XTH11.a. Though XTH proteins identified in this study contained two characteristic conserved domains (Glyco_hydro and XET_C) by searching Pfam database, some XTHs lacked one or several $\alpha$ helices or/and $\beta$-strands compared with PttXET16A. Comparative analysis showed motif 6 covered $\alpha 1$-helices, $\beta 1-\beta 2$ strands, motif 4 covered $\beta 4$, part of $\beta 3$ and $\beta 5$, motif 3 covered $\beta 6$ and part of $\beta 5$, motif 1 covered $\beta 7-$ 8 , motif 2 covered $\beta 9-12$, motif 8 covered $\beta$ 13-14, motif 5 covered $\alpha 1$ and $\beta 15$, respectively. There is no uniform correspondence between motif and $\alpha$-helices or/and $\beta$-strands.

\section{Chromosomal distribution and duplication analysis of $X T H$ genes}

The chromosomal locations of all $X T H$ genes in both Brassica species were investigated based on their physical positions and are shown in Fig. 4. Excluding BraA.XTH1O, which was positioned on a scaffold, the remaining fiftytwo BraXTH genes had definite chromosomal locations; mapping onto the different chromosomes was uneven. Chromosome Ar03 in B. rapa carried the greatest number of genes (13), while Ar04 carried only one $X T H$ gene. In $B$. oleracea, there were $34 X T H$ genes with definite locations and they were distributed among all chromosomes excluding chromosome Co06. Chromosome Co01 was a "hot region", carrying the greatest number of genes (8); in contrast Co04 and Co05 each contained only one XTH gene. Incomplete genome assembly meant that definite chromosomal locations were not available for five XTHs: BraA.XTH10, BolC.XTH2, BolC.XTH27.b, BolC.XTH30.a and BolC.XTH32.c.

TD events contribute to the expansion of gene families and can produce tandemly repeated genes in clusters [47]. We obtained putative tandemly-duplicated XTH genes of the two Brassica species from PTGBase. As a result, 15 $\mathrm{BraXTH}$ genes and $8 \mathrm{BolXTH}$ genes were found to be present in tandem arrays, representing 28.3 and $21.1 \%$ of the total XTH genes in B. rapa and B. oleracea respectively. These tandemly repeated genes were clustered, which was consistent with their chromosomal locations (Fig. 4). Seven tandem arrays were identified on chromosomes Ar01, Ar02, Ar03, Ar08 and Ar010 in B. rapa. Protein BLAST analysis revealed that BraA.XTH17.a is $93 \%$ identical to BraA.XTH17.b, BraA.XTH22C is 99\% identical to BraA.XTH22.c or BraA.XTH22.d, and BraA.XTH22.a is $100 \%$ identical and $75 \%$ coverage to BraA.XTH22.d. The identity of the other tandem gene pairs is varying from 55 to $68 \%$. Four tandem arrays occurred on Co01, Co02, $\mathrm{Co} 03$ and $\mathrm{Co} 07$ in B. oleracea, with 58 to $84 \%$ identity of tandem gene pairs.

In $A$. thaliana, four tandemly duplicated gene arrays composed of nine AtXTHs were found (Fig. 4). 


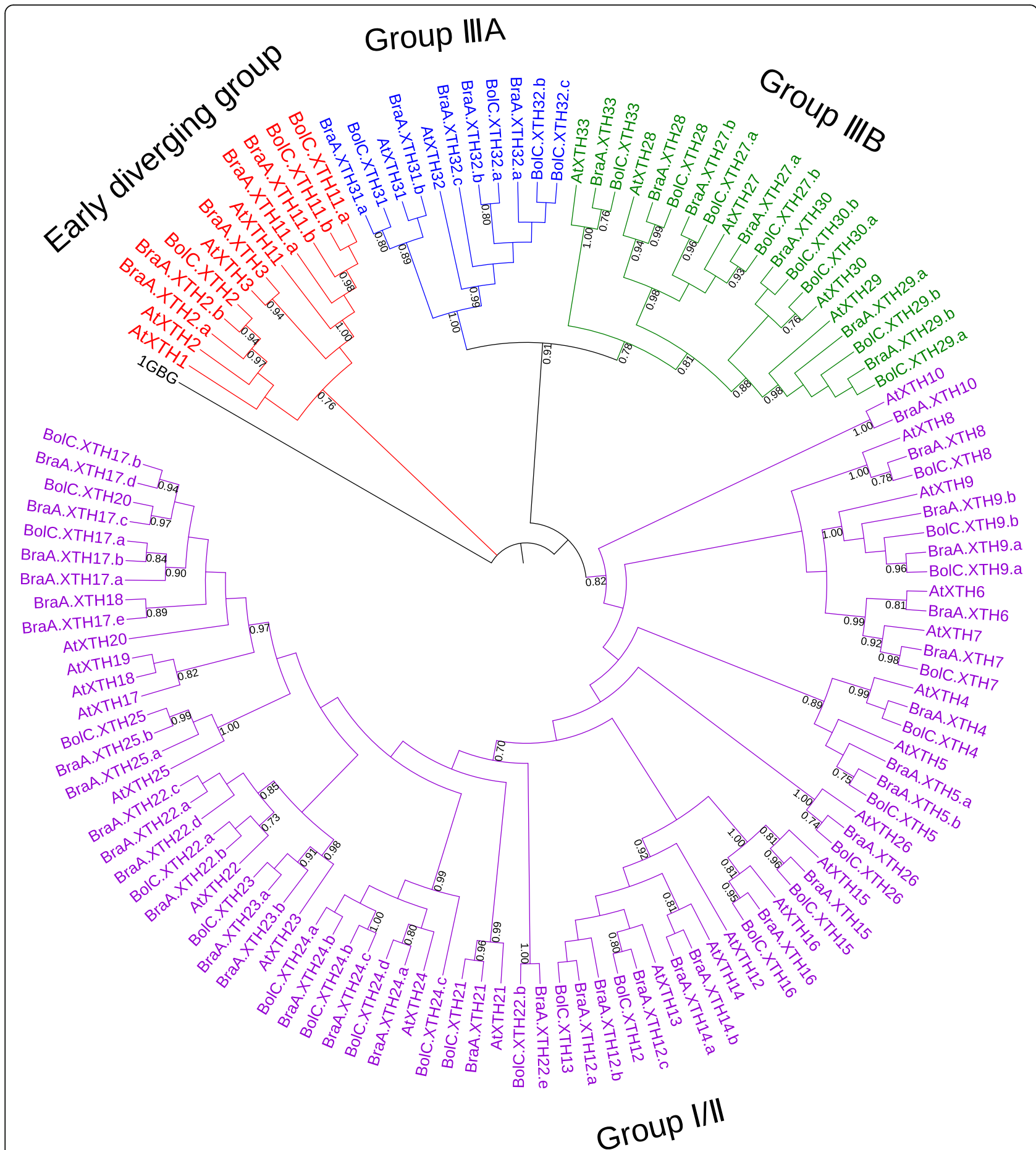

Fig. 1 Phylogenetic analysis of full-length XTH proteins in B. rapa, B. oleracea and Arabidopsis. The tree was constructed by the Maximum Likelihood (ML) method using MEGA7, with the JTT $+G$ model and 1000 bootstrap replicates. The branches correspond to the three phylogenetic groups

Tandem arrays including AtXTH1/2, AtXTH23/14 and AtXTH24/18/19 were located on chromosome At04 while AtXTH12/13/25/22 was on chromosome At05. It is worth mentioning that some genes that bear syntenic relationships to these tandem genes, though not AtXTH1/2, have a conserved tandem repeat pattern in both the $B$. rapa genome and the $B$. oleracea genome, suggesting that these tandem arrays arose before the divergence of $A$. thaliana and the Brassica ancestor. 

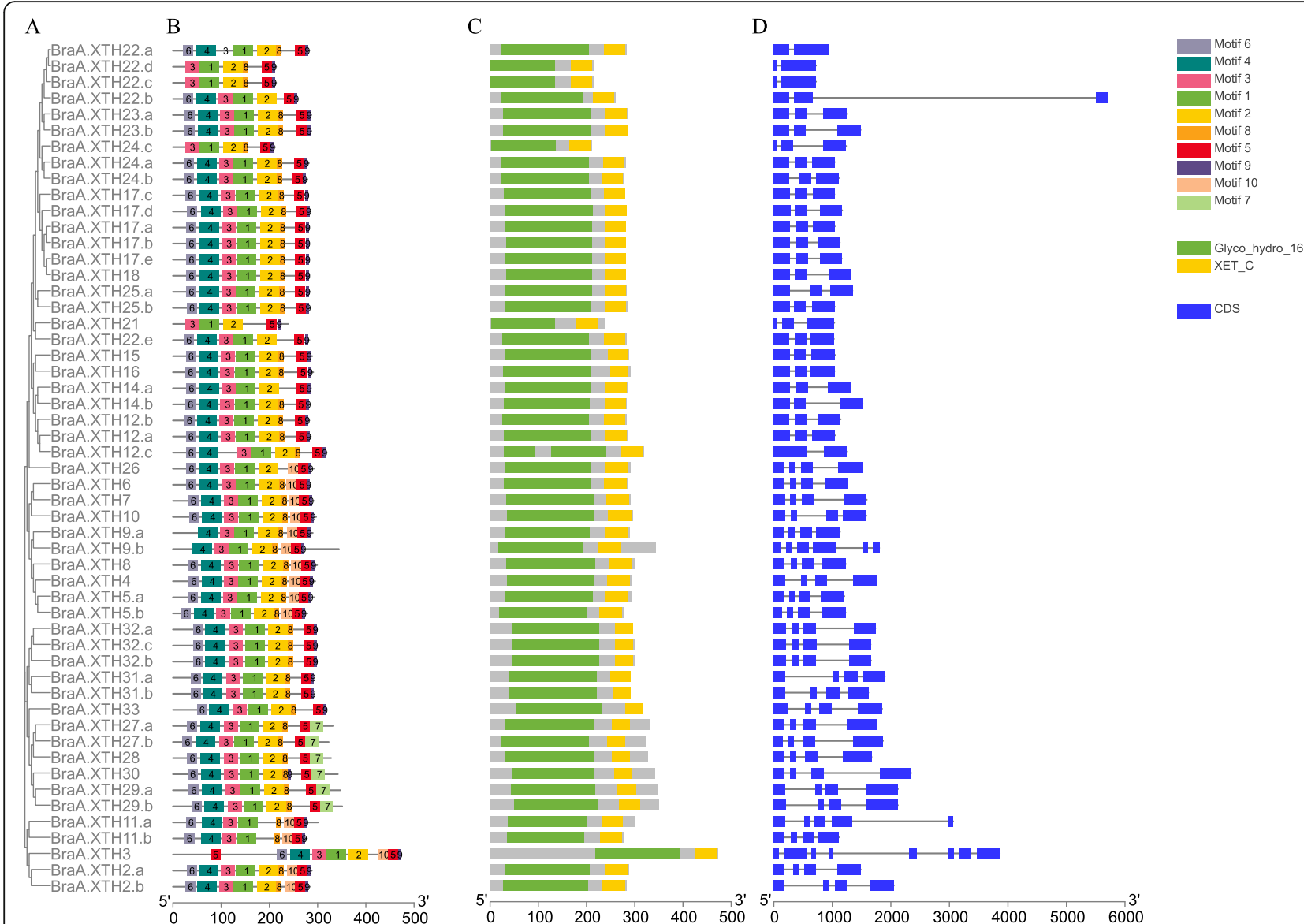

Fig. 2 Characterizations of XTH gene family members in B. rapa, including phylogenetic tree (a), conserved motif location (b), domain location (c) and intron/exon structure (d)

\section{Syntenic analyses of XTH genes}

The ancestor of diploid Brassica species experienced a WGT event since divergence from the Arabidopsis lineage. Syntenic genes are orthologous genes located in fragments syntenic between different species that derive from a shared ancestor, and synteny analysis can be used to transfer gene annotations and investigate genomic evolution in related species [48]. We obtained the genes syntenic with the $X T H$ genes of Arabidopsis for the two Brassica species by searching for 'syntenic gene' in BRAD (Additional file 6). According to comparative genomics analysis, the density and expression level of genes in different regions show some differences in the genomes of $B$. rapa and B. oleracea, which can be divided into three fractionated subgenomes which we denoted LF (Least-fractionated), MF1 (Medium-fractionated), and MF2 (Most-fractionated) according to the extent of gene retention [41, 49]. Statistical analysis indicated that there were 13, 13, and $6 \mathrm{BraXTH}$ genes and 9, 10, and 5 BolXTH genes located in the LF, MF1 and MF2 subgenomes respectively (Additional file 6). In summary, 60.4 and $63.2 \%$ of the total $X T H$ genes in, respectively, $B$. rapa and $B$. oleracea were located in syntenic blocks. WGD events are therefore likely to have played a major role in the expansion of XTH genes in the two Brassica species. The identities of $75 \%$ (24 out of 32) BraXTHs and 62.5\% (15 out of 24) BolXTHs with their Arabidopsis syntenic orthologs exceeded 80\% (Additional file 6).

A total of 23 AtXTH genes had corresponding syntenic genes in the two Brassica species. The copy numbers of syntenic genes in the genomes of the two Brassica species differed. The first situation was one in which genes syntenic with $A t X T H$ genes were completely preserved in the same syntenic block in the Ar and Co subgenome; 8 genes were of this type. In the second case, AtXTH genes were retained in the Ar genome but lost from the Co genome, this applied to AtXTH3 and AtXTH5. The third case was where AtXTH genes had more than one syntenic gene in $B$. rapa or $B$. oleracea. For example, 8 and 1 AtXTH genes had 3 syntenic genes in $B$. rapa and B. oleracea respectively. An AtXTH should theoretically correspond to 3 syntenic genes and if there are fewer than 3 it may be the result of gene loss after genome replication. 

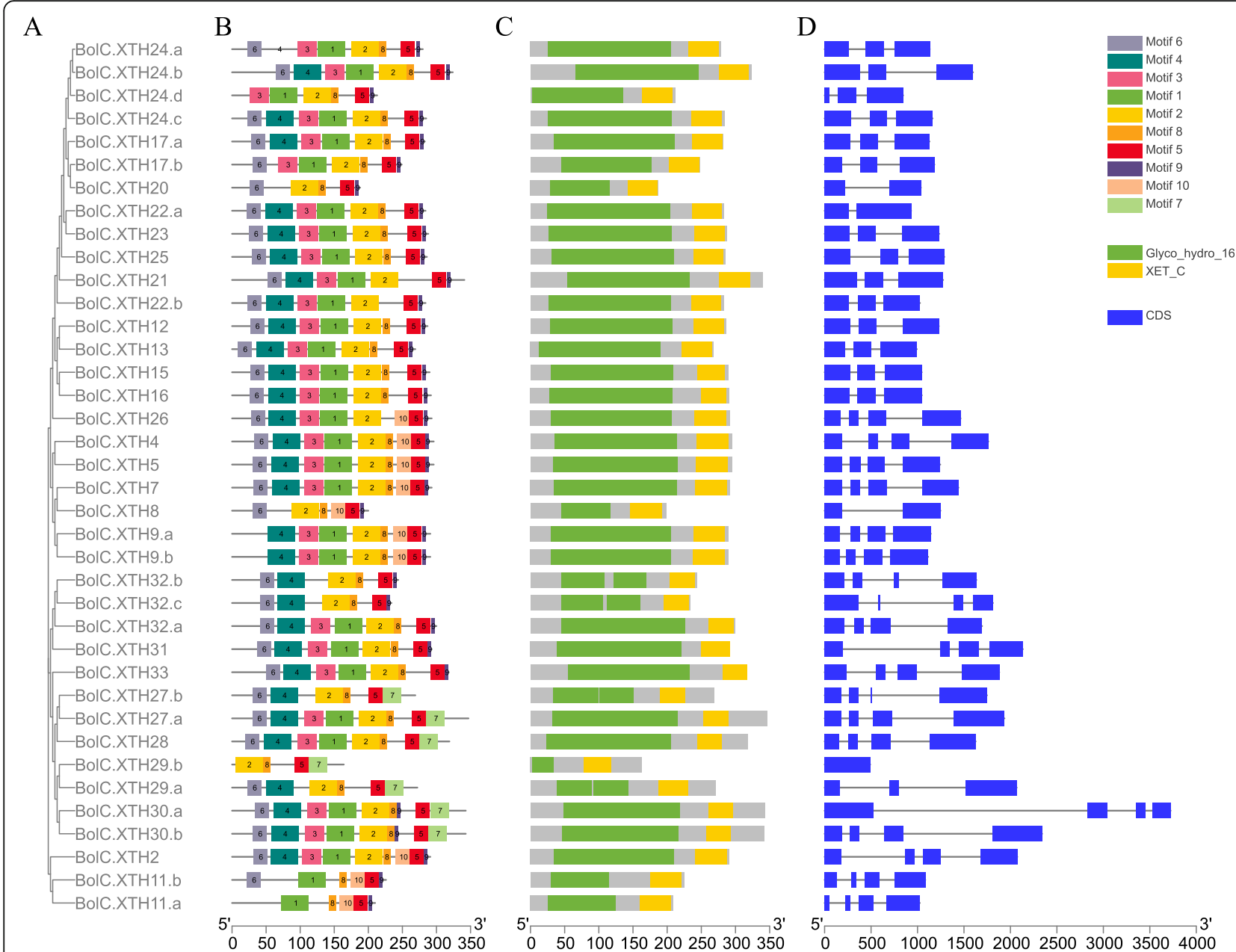

Fig. 3 Characterizations of XTH gene family members in B. olerace, including phylogenetic tree (a), conserved motif location (b), domain location (c) and intron/exon structure (d)

\section{Selection forces acting on XTH duplicated pairs}

To assess whether XTH duplicated pairs in Brassica species experienced different selective forces, $\mathrm{Ka} / \mathrm{Ks}$ values were calculated (Additional file 7). A Ka/Ks ratio $>1$ represents positive selection, $\mathrm{Ka} / \mathrm{Ks}=1$ represents neutral selection and a $\mathrm{Ka} / \mathrm{Ks}$ ratio $<1$ represents purifying selection [50]. We found 33 and 18 segmentally duplicated $\mathrm{XTH}$ gene pairs in B. rapa and B. oleracea respectively. All segmentally duplicated XTH gene pairs had $\mathrm{Ka} / \mathrm{Ks}<$ 1, while two tandemly duplicated gene pairs (BraA.XTH22.a-BraA.XTH22.d and BraA.XTH22.cBraA.XTH22.d) had no $\mathrm{Ka} / \mathrm{Ks}$ value in B. rapa because they shared the same sequence.

The segmental duplications of the XTH genes in $B$. rapa originated between 0.34 Mya $(\mathrm{Ks}=0.0103)$ and 28.80 Mya $(\mathrm{Ks}=0.8640)$, with a mean of 12.88 Mya $(\mathrm{Ks}=0.1436)$. After comparative analysis, the segmental duplications of the BolXTH genes were found to have originated from 5.37 Mya $(\mathrm{Ks}=0.1612)$ to 32.12 Mya $(\mathrm{Ks}=0.9637)$, with a mean of 13.20 Mya $(\mathrm{Ks}=0.3960)$.
Overall, the $\mathrm{Ka} / \mathrm{Ks}$ ratios for segmental duplication of BolC.XTH11.b and BolC.XTH11.a, BraA.XTH2.b and BraA.XTH2.a, together with BraA.XTH23.a and BraA.XTH23.b, were $>0.3$, while the ratios for the other segmental duplication pairs were all $<0.3$, suggesting that significant functional divergence of some XTH genes might have occurred after the duplication events.

\section{Expression patterns of $X T H$ genes in different tissues of $B$.} rapa and $B$. oleracea

To understand the variations in expression pattern for $X T H$ genes, we analyzed $X T H$ gene expression patterns across different tissues in the two species of Brassica based on RNA-Seq retrieved from the GEO database (Additional file 8). If the FPKM of a gene was less than 1 , it was considered to be an unexpressed gene in this study, including BraA.XTH2.a/b, BraA.XTH5.b, BraA.XTH11.a, BraA.XTH12.a/b/c, BraA.XTH25.a/b, BolC.XTH5, BolC.XTH11.a, BolC.XTH20, BolC.XTH21, BolC.XTH22.b, BolC.XTH24.c, BolC.XTH25 and 


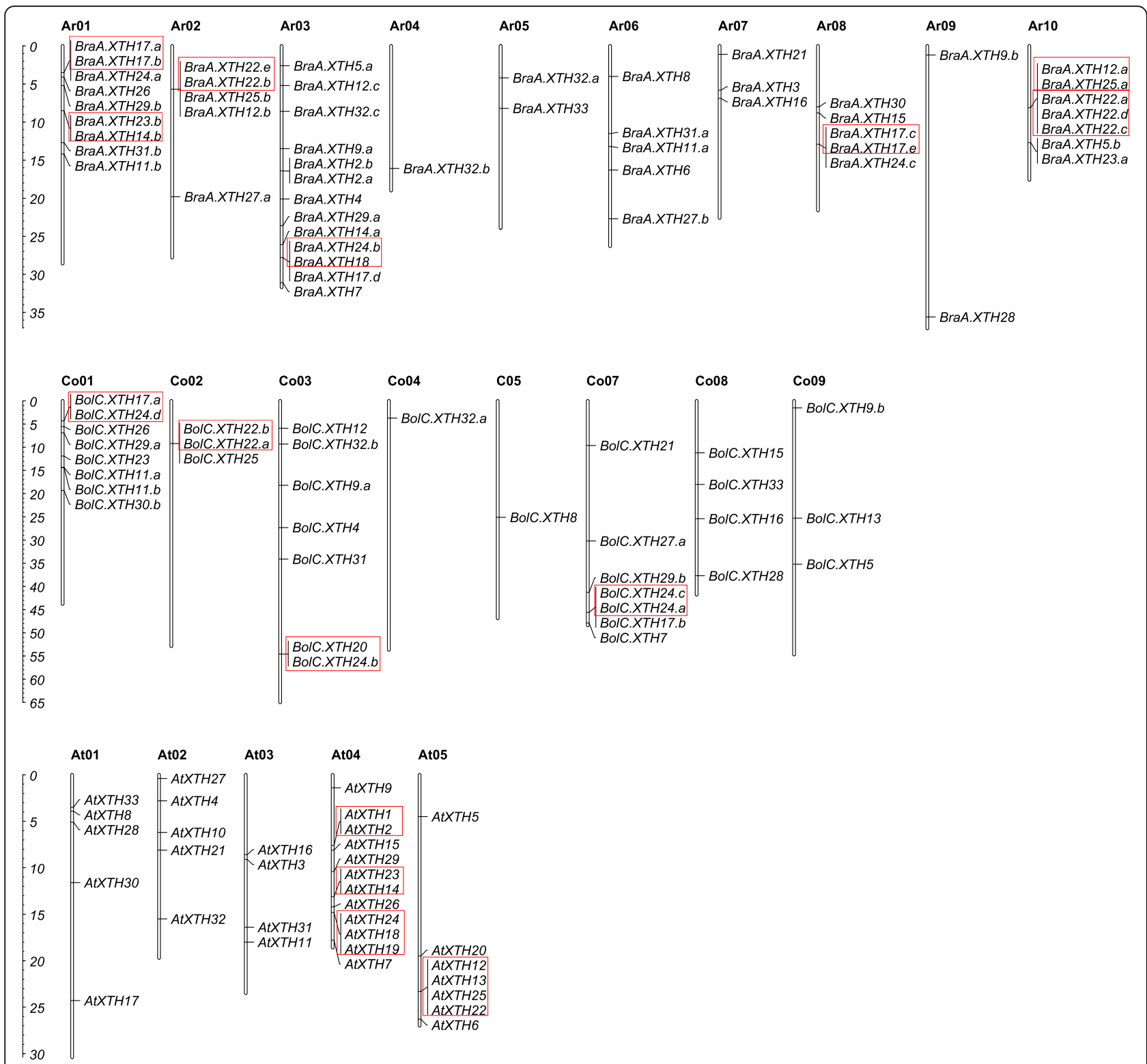

Fig. 4 Distribution of XTH genes on B. rapa, B. oleracea and A. thaliana chromosomes. XTH genes in red boxes are tandemly repeated genes. The number of chromosomes was indicated at the top of each chromosome. Ar, Co and At in front of number represent chromosome in B. rapa, B. oleracea and A. thaliana, respectively. The scale on the left is in megabases (Mb)

BolC.XTH26. In addition, BolC.XTH12 and BolC.XTH13 lacked FPKM values. On this basis, 44 BraXTH genes and $28 \mathrm{BolXTH}$ genes were expressed in at least one tissue, while the remaining genes lacked expression data or were unexpressed in all the tissues tested, indicating that they might be non-functional or have specific temporal and spatial expression patterns that were not detected in this study. There were 23 out of 53 (approximately 43.4\%) BraXTH genes and 14 out of 38 (approximately 36.8\%) BolXTH genes that were widely expressed in all the tissues tested (root, stem, leaf, flower, silique and callus of B. rapa; root, stem, leaf, flower, silique, callus and bud of B. oleracea). The remaining $21 \mathrm{BraXTH}$ genes and 14 BolXTH genes were expressed in at least one but not in all tested tissues. For example, BraA.XTH29.a and BraA.XTH29.b were expressed specifically in the flower; BraA.XTH10, BraA.XTH17.c, BraA.XTH17.d and BraA.XTH32.b were expressed in all tissues except callus. BolC.XTH2 was expressed solely in the silique and BolC.XTH29.b was expressed only in buds at low levels.

Clustering analysis of expression values showed that both the $B$. rapa and the $B$. oleracea $X T H$ genes can 
be divided into four groups (Fig. 5). In B. rapa, XTH genes in cluster 1 were more highly expressed in the leaf than in the other tissues examined, while cluster 2 were expressed mainly in the root, apart from BraA.XTH32.b and BraA.XTH9.b. Cluster 3 showed higher expression in callus and group 4 was expressed mainly in flower, silique or callus. In $B$. oleracea, $X T H$ genes in cluster 1 were highly expressed in the root, whereas cluster 2 was expressed mainly in the flower. Four genes in cluster 3 were expressed mainly in the stem or leaf and genes in cluster 4 were expressed mainly in the leaf, silique or callus. XTH genes in the same group based on phylogenetic analysis did not show the same expression patterns.

Some tandemly repeated family members, such as BraA.XTH22.a and BraA.XTH22.c in cluster 1, showed similar expression patterns across the tissues tested, indicating the possible existence of redundancy (Fig. 5a). However, most tandemly repeated members displayed distinct expression patterns. For example, BolC.XTH24.a and BolC.XTH24.b showed higher expression levels in the flower than the other tissues, whereas tandem repeats of them, BolC.XTH24.c and BolC.XTH20, were not expressed in these tissues. BolC.XTH17.a showed high expression in the root and low expression in the bud, leaf and silique, while BolC.XTH24.d showed high expression in the flower and low expression in the leaf (Fig. 5, Additional file 8). All $X T H$ tandem genes in seven arrays were also analyzed and compared in B. rapa. A total of 2 tandem genes (BraA.22b/e and BraA.14b/23b) showed different abundances, but the same trend with respect to patterns, whereas the two members of each of the other pairs of tandem genes showed differences in abundance and tissue specificity of expression. In general, $X T H$ genes in the two Brassica species exhibit differential patterns of expression across different tissues, leading to different functional clusters and suggesting functional divergence.

\section{Discussion}

The $X T H$ gene family expanded in $B$. rapa and $B$. oleracea Previous studies revealed that the Brassica genome, like that of $A$. thaliana, underwent three paleopolyploidy events. Brassica species also shared an additional WGT event since isolation from Arabidopsis [40, 41]. Compared to the 33 AtXTH genes [13], higher numbers of $X T H$ genes were identified in the $B$. rapa (53 genes) and $B$. oleracea (38) genomes. Moreover, 60.4 and $63.2 \%$ of the total XTH genes in, respectively, $B$. rapa and $B$. oleracea were located in syntenic blocks. WGD events therefore played a major role in the expansion of XTH genes in these two Brassica species. The secondary force leading to the expansion of XTH genes was tandem duplication.
There were, respectively, 28.3 and $21.1 \%$ of the total $X T H$ genes that were involved in tandem arrays in $B$. rapa and $B$. oleracea, and $B$. rapa has more tandem $X T H$ genes than $B$. oleracea, which indicated that $B$. oleracea has lost some of its XTH tandem genes during the process of gene duplication. It reflects the amplification of tandem repeat genes is asymmetric between the two species. Tandem duplication also contributed to $X T H$ gene family expansion in barley, tobacco, sorghum, and soybean $[15,19,51,52]$.

Since the Brassica ancestor diverged from its common ancestor with $A$. thaliana $\sim 20$ Mya; it subsequently underwent a WGT event $\sim 15.9$ Mya. Then the Brassica ancestor diverged to form the modern $B$. rapa and $B$. oleracea about 3.75 Mya [35-38]. In this study, we found that most of the segmental duplications of BraXTH and BolXTH genes occurred before the divergence of the modern $B$. rapa and $B$. oleracea. However, BraA.XTH16 and BraA.XTH15 arose around 28.80 Mya before the divergence of the Brassica ancestor and its common ancestor with A. thaliana, while BraA.XTH23.a and BraA.XTH23.b arose around 0.34 Mya after the divergence of the modern B. rapa and B. oleracea.

\section{The XTH gene family is highly conserved at the DNA and protein level}

The XTH proteins of A. thaliana $B$. rapa and B. oleracea can be divided into 3 groups according to the results of phylogenetic analysis. The number of genes in Group IIIA is the smallest, while the number in Group I/II is the largest, which is consistent with results obtained from other plants [20]. All XTH proteins were found to be located at the cell walls and this positioning is consistent with the function of XTH proteins involved in cell wall reconstruction. In addition, some XTHs, all members of Group I/II, were also found to be located in the cytoplasm. These findings were similar to those reported in barley and pineapple $[15,20]$. Interestingly, the TD genes all belong to Group I/II, suggesting that TD is the reason for the large number of genes in this group. It has been reported that proteins showing XET activity belong mostly to Groups I/II and IIIB, while proteins showing XEH activity belong mostly to group IIIA [3, 9, 26]. Thus Brassica XTH members in different groups may show different types of enzyme activity. Unlike the proteins in other groups, the Group IIIB proteins of the two species contained motif 7 , suggesting that this motif may be related to the specific function(s) of the IIIB proteins.

\section{Gene and domain loss events in the XTH family}

The genome size of B. rapa and B. oleracea is different, at about $529 \mathrm{Mb}$ and $696 \mathrm{Mb}$ respectively, and the total number of genes according to the PLAZA 4.5 database 

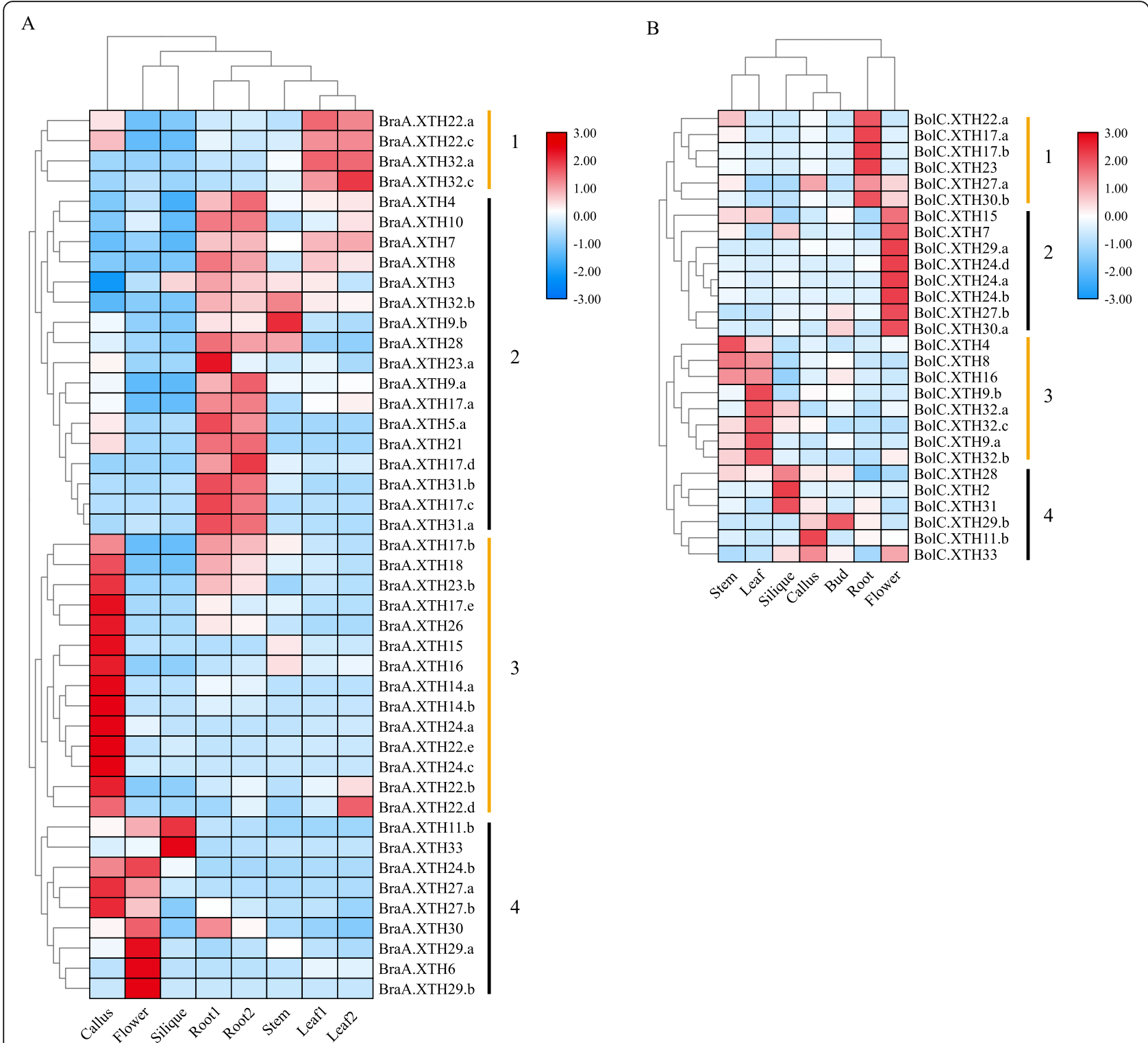

Fig. 5 Heatmaps of expression clustering for XTH genes across different tissues in B. rapa (a) and B. oleracea (b) based on FPKM values retrieved from the GEO database (http://www.ncbi.nlm.nih.gov/geo/). Two samples of root and leaf tissues were generated from different batches of plants for Fig. 5a. Color scale bars representing relative signal ratios are shown at the top of the right-hand side of each heatmap. Heatmaps were drawn with TBtools. The relative level of expression of a particular gene in each row was normalized against the mean value. Euclidean distance was used to evaluate closeness between genes, and the average linkage method was used for cluster analysis

is about 42,000 and 45,000 respectively [27]. However, $B$. rapa apparently has more $X T H$ s than $B$. oleracea; this may be because the $B$. oleracea genome assembly was incomplete, leading to incomplete identification, or may be due to a greater loss of XTH genes from $B$. oleracea.

Compared with A. thaliana, the Brassica genome experienced a unique triplication event [40, 41]. Hence, each XTH gene in Arabidopsis should correspond to three homologs in B. rapa and B. oleracea. However, the number of $X T H$ genes obtained from each of the two species was far less than three times the number of $X T H$ genes in A. thaliana. All the AtXTH genes had $0-2$ orthologs in the genomes of the two species, apart from AtXTH32, which had three homologs. This indicates that the loss of XTH genes occurred after the Brassica WGT event. Synteny analysis revealed that $61.5 \%$ of the XTH genes of the two Brassica species were located in conserved chromosomal blocks, whereas some genes had been deleted. These syntenic blocks account for the majority of the XTH genes in A. thaliana $(75.76 \%$ of the genes), $B$. oleracea (63.16\%) and B. rapa (84.21\%). At the whole genome 
level syntenic blocks contain 72.24, 57.88 and $64.84 \%$ of the genes in A. thaliana, B. oleracea and B. rapa respectively [41]. $B$. oleracea had lost a considerably greater number of $X T H$ genes compared with B. rapa, consistent with the asymmetry of gene loss between the two genomes.

After carrying out comparative analysis of the pattern of retention/loss of orthologous genes in each set of three subgenomic (LF, MF1 and MF2) blocks of the two species corresponding to $A$. thaliana, we found the $X T H$ genes retained in $B$. rapa and $B$. oleracea are mostly located in the LF subgenome and the MF1 subgenome, which is consistent with the retention pattern for their genomes as a whole. The MF2 subgenome retained the fewest $X T H$ genes and has thus undergone the greatest loss of orthologous genes. The MF1 subgenome had lost the largest number of genes at the whole genome level. There were differences in the levels of XTH loss among the three subgenomic (LF, MF1 and MF2) blocks, which was consistent with the difference in gene loss rate among subgenomes [41].

Previous research demonstrated that statistically, more than one-third of all domains have a marked tendency to increase/decrease in size during protein evolution [53]. XTH proteins generally contain a characteristic motif, EXDXE, which contains amino acid residues that mediate catalytic activity. Sitedirected mutation of AtXTH22 has indicated that the first glutamine residue in this motif is required for catalytic activity [54]. Compared with the AtXTH protein structures, there were seven BolXTHs lacking the EXDXE conserved active-site motif: BolC.XTH8, BolC.XTH20, BolC.XTH27.b, BolC.XTH29alb and BolC.XTH32.b\c. In addition, several proteins encoded by syntenic XTH genes had a Glyco_hydro_16 domain but lacked an XET_C domain, so that they could not be identified as XTHs in this study. These phenomena reflect differences in the evolution of homologous genes between the $B$. rapa genome and the $B$. oleracea genome, and the higher level of DNA loss from the $B$. oleracea genome. Domain loss has also been observed in Hsp70 genes of Brassica species [55].

\section{The patterns of expression of $X T H$ genes}

Previous research using GUS staining in Arabidopsis [56] showed that AtXTHs are probably expressed in all developmental stages from seed germination through to flowering. In this study, 83 and $74 \%$ of the $X T H$ genes in, respectively, $B$. rapa and $B$. oleracea were expressed across all the tissues examined. Comparative analysis showed that the AtXTH orthologs in B. rapa and B. oleracea showed different expression characteristics, even among orthologous genes with high levels of identity of amino acid sequences. In a previous study AtXTH21,
AtXTH22 and AtXTH3O were found to be expressed mainly in siliques [13]. However, their orthologs in the two Brassica species exhibited different expression patterns. For example, BraA.XTH21 had only a low expression level in callus and roots, but BolC.XTH21 was not express; BolC.XTH22.a was expressed mainly in roots, stems and callus, while BolC.XTH22.b was not expressed in any of the tissues examined; BraA.XTH3O showed high expression levels in callus, roots and flowers, while BolC.XTH30.a showed its highest expression level in flowers, and BolC.XTH30.b was expressed most highly in roots. BraA.XTH33 showed the highest expression level in siliques, consistent with its Arabidopsis homolog (AtXTH33), but BolC.XTH33 was expressed in callus, silique and flower at an intermediate level. BolC.XTH24.al $b / d$ all showed the highest expression levels in flowers but BolC.XTH24.c showed no expression in this tissue, BraA.XTH24.a/b/c showed their highest expression levels in callus, followed by flowers, whereas the expression of its Arabidopsis homolog (AtXTH24) was mainly in stems [13].

Duplicated genes usually share high levels of sequence similarities; however, over the course of evolution, the fates of duplicated genes may be quite different, as they undergo nonfunctionalization, neofunctionalization or subfunctionalization [57]. As a result of comparative analysis of the expression profiles of $X T H$ genes involved in tandem and segmental duplications, patterns of similar, different or silenced gene expression relative to other members were found. For example, BraA.XTH32. $a$ and BraA.XTH32.c, BraA.XTH14.a and BraA.XTH14.b, which were segmentally duplicated genes, exhibited similar expression behavior, indicating that their roles may have been conserved after the duplication events. BraA.XTH23.a and BraA.XTH23.b, together with BolC.XTH11.a and BolC.XTH11.b, showed different expression patterns, suggesting that divergence in gene expression may have been associated with the acquisition of novel characteristics (Fig. 5, Additional file 8). BraA.XTH5.a was expressed in roots but BraA.XTH5.b was not expressed in any of the tissues tested, suggesting that it had experienced nonfunctionalization after the duplication events (Fig. 5a, Additional file 8).

\section{Conclusions}

In this study, 53 and $38 X T H$ genes were identified in $B$. rapa and $B$. oleracea respectively. They, together with the 33 Arabidopsis XTH genes, were classified into three groups (Early diverging group, Group I/II and Group III) by phylogenetic analysis based on clade support values, the topology of the phylogenetic tree, and the previous classification of XTH families in Arabidopsis. Exonintron distribution and comparisons of conserved motifs also supported this classification of XTH genes. Analysis 
of expansion mechanisms revealed that a WGT event exerted the most major influence, followed by TD events, on the expansion of the $X T H$ gene family in both Brassica species. Gene loss events have occurred in the $X T H$ gene family in the two species; the extent of loss was greater in B. oleracea than in B. rapa. RNA-seq data analysis provided insight into species-specific functional divergence among members of the $X T H$ gene family. Taken together, these results increase our understanding of the evolution of the $X T H$ gene family and provide a reference for future determination of the functions of each $X T H$ gene across Brassica species.

\section{Methods}

\section{Data sources}

Genomic sequences, CDS sequences, protein sequences and annotation information for $B$. rapa and B. oleracea were downloaded from the BRAD database (http://brassicadb.org) [58]. A. thaliana XTH protein sequences were downloaded from TAIR (http://www.arabidopsis. org/) [59].

Identification of XTH genes and analysis of their characteristics

Three methods were used to identify XTH proteins in this study. First, all 33 A. thaliana XTH sequences obtained from the TAIR database were used as query sequences to carry out a BLASTp (E-value <1e-5) search for all protein sequences from $B$. rapa and $B$. oleracea in the BRAD database. Second, the HMM profiles of the Glyco_hydro_16 domain (PF00722) and XET_C domain (PF06955) were obtained from the Pfam database (https://Pfam.xfam.org/) [60] and used to search all $B$. rapa and $B$. oleracea proteins with the HMM search tool in the TBtools software package [58] with default parameters. The third approach was to search for syntenic genes in the BRAD database by inputting the $A$. thaliana $\mathrm{XTH}$ gene IDs [61]. After integrating the results of the three methods, all redundant sequences were removed manually, after which candidate XTH protein sequences were filtered using the CDD tool (http://www.ncbi.nlm. nih.gov/Structure/cdd/wrpsb.cgi/) [62]. Only proteins that contained both the Glyco_hydro_16 domain and the XET_C domain were regarded as XTHs and reserved for further analysis. Finally, all genes identified as encoding $\mathrm{XTH}$ proteins were designated with reference to a previous study [63].

The molecular weight $(\mathrm{Mw})$, number of amino acids and theoretical isoelectric point $(\mathrm{pI})$ of each protein were obtained from ProtParam (http://web.expasy.org/protparam/) [64]. Plant-mPLoc (http://www.csbio.sjtu.edu. cn/bioinf/plant-multi/) [65] was used to predict patterns of protein subcellular localization. The TargetP-2.0 Server (http://www.cbs.dtu.dk/services/TargetP/) [66] was used to predict the presence or absence of signal peptides. The MEME tool (http://meme-suite.org/tools/ meme) [67] was employed to predict and analyze the motifs in each protein, with parameters set as follows: motif width 6-60, maximum number of motifs 10 and default values were used for the remaining parameters. The distribution of motifs was illustrated using the Redraw motif pattern tool in TBtools.

\section{Comparative phylogenetic analysis of XTH proteins}

Multiple sequence alignments of the full-length XTH protein sequences from B. rapa, B. oleracea and $A$. thaliana were performed using Clustal X1.8, then MEGA7 was used to analyze the results (Additional file 9) [68]. The phylogenetic tree was constructed based on the Maximum Likelihood (ML) method, with the JTT + G model and 1000 bootstrap replicates. The phylogenetic tree was visualized using the iTOL online tool (https:// itol.embl.de/) [69].

\section{Structural-based sequence alignment}

ESPript (http://espript.ibcp.fr/ESPript/ESPript/) [70] was used to predict the secondary structures as well as the presence of structural elements in the XTH protein sequences. The crystal structure of PttXET16 (PDB id: 1UN1) [53] was obtained from the PDB databank to locate secondary structures.

\section{Analysis of chromosomal locations and gene duplication} The chromosomal locations of $X T H$ genes were derived from BRAD. All XTH genes were mapped to chromosomes by MapChart [71] except for a few that were located on unassigned scaffolds. Duplicated XTH gene pairs were identified by the BLASTn program with both coverage and identity set to $>80 \%$ in the two species [72]. Putative tandemly-duplicated genes in A. thaliana, $B$. rapa, and $B$. oleracea were retrieved from PTGBase (http://ocri-genomics.org/PTGBase) [73].

To estimate the modes of selection acting on $X T H$ genes, the $\mathrm{Ka} / \mathrm{Ks}$ ratios between duplicated $X T H$ gene pairs were calculated. $\mathrm{Ka} / \mathrm{Ks}$ ratios greater than 1 , less than 1 , and equal to 1 represented positive selection, negative selection, and neutral selection respectively [74]. For each gene pair, the Ks value was used to estimate the divergence time $(\mathrm{T})$ as millions of years ago based on a rate of $1.5 \times 10^{-8}$ substitutions per site per year, using the formula $\mathrm{T}=\mathrm{Ks} /\left(2 \times 1.5 \times 10^{-8}\right)$ Mya [75].

\section{Analysis of tissue expression patterns}

RNA-seq expression data for B. rapa tissues (GSE43245) and $B$. oleracea tissues (GSE42891) were downloaded from the GEO database at NCBI (http://www.ncbi.nlm. nih.gov/geo/) [41, 76]. The XTH expression data were measured using the expressed FPKM values for transcripts assembled and analyzed in a previous study [77, 
78]. FPKM values for different tissue were subjected to hierarchical clustering analysis with TBtools. The data were normalized in order to more intuitively examine differences in expression of the same gene in different samples and represented as a heatmap with TBtools; genes with FPKM values of less than one in all samples were not included in the heatmap.

\section{Ethics approval}

This article does not contain any studies with human participants or animals performed by any of the authors.

\section{Supplementary Information}

Supplementary information accompanies this paper at https://doi.org/10. 1186/s12864-020-07153-1.

Additional file 1. XTHs in B. rapa and B. oleracea with their Arabidopsis orthologs.

Additional file 2. The best-hits between BraXTHs in this study and BraXTHs identified in previous report.

Additional file 3. The ML resulting phylogeny in Nexus format.

Additional file 4. Structure-based sequence alignment of BraXTHs and PttXET16A. Sequences were aligned using ClustalX and generated by ESPript [70]. The secondary structure elements indicated above the alignment are those of PttXET16A, Populus tremula $\times$ tremuloides XET16A (AF515607), whose structure has been experimentally determined [45]. Blue frames indicate conserved residues, white letters in red boxes indicate strict identity, and red letters in white boxes indicate similarity. The predicted $a$-helices and $\beta$-strands are represented by spirals and horizontal arrows, respectively.

Additional file 5. Structure-based sequence alignment of BolXTHs and PttXET16A. Sequences were aligned using ClustalX and generated by ESPript [70]. The secondary structure elements indicated above the alignment are those of PttXET16A, Populus tremula $\times$ tremuloides XET16A (AF515607), whose structure has been experimentally determined [45]. Blue frames indicate conserved residues, white letters in red boxes indicate strict identity, and red letters in white boxes indicate similarity. The predicted $a$-helices and $\beta$-strands are represented by spirals and horizontal arrows, respectively.

Additional file 6. XTH genes syntenic in A. thaliana, B. rapa and B. oleracea.

Additional file 7. $\mathrm{Ka} / \mathrm{Ks}$ analysis and estimated time of divergence of duplicated pairs of XTH genes in B. rapa and B. oleracea.

Additional file 8. FPKM values for XTH genes in different tissues of $B$. rapa and B. oleracea.

Additional file 9. The alignment file used as input for the ML analysis in FASTA format.

\footnotetext{
Abbreviations

A. thaliana: Arabidopsis thaliana; B. oleracea: Brassica oleracea; B. rapa: Brassica rapa; BLASTp: Basic Local Alignment Search Tool; FPKM: Fragments per kilobase of transcript per million fragments sequenced; Ka: Non-synonymous substitution; Ks: Synonymous substitution; MW: Molecular weight; MYA: Million years ago; NJ: Neighbor-joining; XTH: Xyloglucan endotransglucosylase/hydrolase; LF: Least fractionated; MF1: Medium fractionated; MF2: Most fractionated; WGD: Whole genome duplication; WGT: Whole genome triplication; TD: Tandem duplication; HMM: Hidden Markov Model
}

\section{Acknowledgments}

Not applicable.

\section{Authors' contributions}

DW analyzed the influence of WGD and TD for XTH gene family, Ka/Ks value and estimated divergence timed of duplication XTH gene pairs, as well as expression analysis of XTH gene family, prepared the manuscript. AQL identified the members of XTH genes and finished the phylogenetic analysis of XTH gene family. XYQ analyzed the characteristics of XTHs and sequence alignment of XTHs. JYL analyzed the motif, domain, gene structure and syntenic genes. MS supervised the project and revised the manuscript. All authors read and approved the final manuscript.

\section{Funding}

This work was supported by funding to Min Song from the Science and Technology project of Shandong Education Department (Grant no. J15LE02) and the China Postdoctoral Science Foundation funded project (Grant no. 2018 M632646). The funding body did not participate in the design of the study, collection, analysis and interpretation of data or in writing the manuscript.

\section{Availability of data and materials}

All data generated or analyzed during this study are included in this published article and the additional files.

Ethics approval and consent to participate

Not applicable.

\section{Consent for publication}

Not applicable.

\section{Competing interests}

The authors declare that they have no competing interests.

Received: 18 August 2020 Accepted: 14 October 2020

Published online: 11 November 2020

\section{References}

1. Xuan Y, Zhao HF, Guo XY, Ren J, Wang Y, Lu BY. Plant Cell Wall remodeling enzyme Xyloglucan Endotransglucosylase/hydrolase (XTH). Chin Agric Sci. Bull. 2016;18:83-8.

2. Thompson JE, Fry SC. Restructuring of wall-bound xyloglucan by transglycosylation in living plant cells. Plant J. 2001;26:23-34.

3. Fry SC, Smith RC, Renwick KF, Martin DJ, Hodge SK, Matthews KJ. Xyloglucan endotransglucosylase, a new wall-loosening enzyme activity from plants. Biochem J. 1992;282:821-8.

4. Strohmeier M, Hrmova M, Fischer M, Harvey AJ, Fincher GB, Pleiss J. Molecular modeling of family GH16 glycoside hydrolases: potential roles for xyloglucan transglucosylases/hydrolases in cell wall modification in the poaceae. Protein Sci. 2004;13:3200-13.

5. Viborg AH, Terrapon N, Lombard V, Michel G, Czizek M, Henrissat B, et al. A subfamily roadmap of the evolutionarily diverse glycoside hydrolase family 16 (GH16). J Biol Chem. 2019. https://doi.org/10.1074/jbcRA119010619.

6. Baumann MJ, Eklof JM, Michel G, Kallas AM, Teeri TT, Czjzek M, et al. 3rd structural evidence for the evolution of xyloglucanase activity from xyloglucan endo-transglucosylases: biological implications for cell wall metabolism. Plant Cell. 2007;19:1947-63.

7. Rose JK, Braam J, Fry SC, Nishitani K. The XTH family of enzymes involved in xyloglucan endotransglucosylation and endohydrolysis: current perspectives and a new unifying nomenclature. Plant Cell Physiol. 2002;43:1421-35.

8. Eklof JM, Brumer $\mathrm{H}$. The XTH gene family: an update on enzyme structure, function, and phylogeny in xyloglucan remodeling. Plant Physiol. 2010;153: 456-66.

9. Behar H, Graham SW, Brumer H. Comprehensive cross-genome survey and phylogeny of glycoside hydrolase family 16 members reveals the evolutionary origin of EG16 and XTH proteins in plant lineages. Plant J. 2018:95:1114-28.

10. Michailidis G, Argiriou A, Darzentas N, Tsaftaris A. Analysis of xyloglucan endotransglucosylase/hydrolase (XTH) genes from allotetraploid (Gossypium hirsutum) cotton and its diploid progenitors expressed during fiber elongation. J Plant Physiol. 2009;166:403-16.

11. Yokoyama R, Yohei, Harada T, Hiwatashi Y, Hasebe M. Biological implications of the occurrence of 32 members of the XTH (xyloglucan 
endotransglucosylase/hydrolase) family of proteins in the bryophyte Physcomitrella patens. Plant J. 2010;64(4):645-56.

12. Jiao C, Sørensen I, Sun X, Sun H, Behar H, Alseekh S, et al. The Penium margaritaceum genome: hallmarks of the origins of land plants. Cell. 2020; 181(5):1-15

13. Yokoyama R, Nishitani KA. Comprehensive expression analysis of all members of a gene family encoding cell-wall enzymes allowed us to predict cis-regulatory regions involved in cell-wall construction in specific organs of Arabidopsis. Plant Cell Physiol. 2001;42:1025-33.

14. Yokoyama R, Rose JK, Nishitani KA. Surprising diversity and abundance of xyloglucan endotransglucosylase/hydrolases in rice. Classification and expression analysis. Plant Physiol. 2004;134:1088-99.

15. Fu MM, Liu C, Wu F. Genome-wide identification, characterization and expression analysis of Xyloglucan Endotransglucosylase/hydrolase genes family in barley (Hordeum vulgare). Molecules. 2019;24:1935.

16. Geisler L, Geisler M, Coutinho PM, Segerman B, Nishikubo N, Takahashi J, et al. Poplar carbohydrate-active enzymes. Gene identification and expression analyses. Plant Physiol. 2006;140:946-62.

17. Miedes E, Lorences EP. Xyloglucan endotransglucosylase/hydrolases (XTHs) during tomato fruit growth and ripening. J Plant Physiol. 2009; 166:489-98.

18. Song L, Valliyodan B, Prince S, Wan J, Nguyen HT. Characterization of the XTH gene family: new insight to the roles in soybean flooding tolerance. Int J Mol Sci. 2018;19:2705.

19. Wang $M, X u Z$ Z, Ding A, Kong Y. Genome-wide identification and expression profiling analysis of the Xyloglucan Endotransglucosylase/hydrolase gene family in tobacco (Nicotiana tabacum L). Genes. 2018;9:273.

20. Li QY, Li HY, Yin CY, Wang XT, Jiang Q, Zhang R, et al. Genome-wide identification and characterization of Xyloglucan Endotransglucosylase/ hydrolase in Ananas comosus during development. Genes. 2019;10:537.

21. Hyodo H, Yarnakawa S, Takeda Y, Tsuduki M, Yokota A, Nishitani K, et al. Active gene expression of a xyloglucan endotransglucosylase/ hydrolase gene, XTH9, in inflorescence apices is related to cell elongation in Arabidopsis thaliana. Plant Mol Biol. 2003;52:473-82.

22. Vissenberg K, Van Sandt V, Fry SC, Verbelen JP. Xyloglucan endotransglucosylase action is high in the root elongation zone and in the trichoblasts of all vascular plants from Selaginella to Zea mays. J Exp Bot. 2003:54:335-44.

23. Harada T, Torii Y, Morita S, Onodera R, Hara Y, Yokoyama R, et al. Cloning, characterization, and expression of xyloglucan endotransglucosylase/ hydrolase and expansion genes associated with petal growth and development during carnation flower opening. J Exp Bot. 2011;62:815-23.

24. Atkinson RG, Johnston SL, Yauk YK, Sharma NN, Schröder R. Analysis of xyloglucan endotransglucosylase/hydrolase (XTH) gene families in kiwifruit and apple. Postharvest Biol Technol. 2009:51:149-57.

25. Opazo MC, Figueroa CR, Henriquez J, Herrera R, Bruno C, Valenzuela PD, Moya-Leon MA. Characterization of two divergent CDNAs encoding xyloglucan endotransglucosylase/hydrolase (XTH) expressed in Fragaria chiloensis fruit. Plant Sci. 2010:179:479-88.

26. Xuan $Y$, Zhou ZS, Li HB, Yang ZM. Identification of a group of XTHs genes responding to heavy metal mercury, salinity and drought stresses in Medicago truncatula. Ecotoxicol Environ Saf. 2016;132:153-63.

27. Shin Y, Yum H, Kim ES, Cho H, Gothandam KM, Hyun J, et al. BCXTH1, a Brassica campestris homologue of Arabidopsis XTH9 is associated with cell expansion. Planta. 2006;224:32-41.

28. Lee J, Bums TH, Light G, Sun Y, Fokar M, Kasukabe KF, et al. Xyloglucan endotransglucosylase/hydrolase genes in cotton and their role in fiber elongation. Planta. 2010;232:1191-05.

29. Cho SK, Kim JE, Park JA, Eom TJ, Kim WT. Constitutive expression of abiotic stress-inducible hot pepper CaXTH3, which encodes a xyloglucan endotransglucosylase/hydrolase homolog, improves drought and salt tolerance in transgenic Arabidopsis plants. FEBS Lett. 2006;580:3136-44.

30. Choi JY, Seo YS, Kim SJ, Kim WT, Shin JS. Constitutive expression of CaXTH3, a hot pepper xyloglucan endotransglucosylase / hydrolase, enhanced tolerance to salt and drought stresses without phenotypic defects in tomato plants (Solanum lycopersicum cv. Dotaerang). Plant Cell Rep. 2011;30: $867-77$

31. Han YS, Sa G, Sun J, Shen Z, Zhao R, Ding M, et al. Overexpression of Populus euphratica xyloglucan endo-transglucosylase/hydrolase gene confers enhanced cadmium tolerance by the restriction of root cadmium uptake in transgenic tobacco. Environ Exp Bot. 2014;100:74-83.
32. Han YS, Wang W, Sun J, Ding MQ, Zhao R, Deng SR, et al. Polus euphratica XTH overexpression enhances salinity tolerance by the development of leaf succulence in transgenic tobacco plants. J Exp Bot. 2013;64:4225-38.

33. Chen JR, Chen YB, Ziemianska M, Liu R, Niedźwiecka-Filipiak I, Li YL, et al. Co-expression of MtDREBIC and RCXET enhances stress tolerance of transgenic China rose (Rosa chinensis Jacq). J Plant Growth Regul. 2016;35: $586-99$.

34. Zhu XF, Shi YZ, Lei GJ, Fry SC, Zhang BC, Zhou YH, et al. XTH37, encoding an in vitro XEH/XET-active enzyme, regulates aluminum sensitivity by modulating in vivo XET action, cell wall xyloglucan content, and aluminum binding capacity in Arabidopsis. Plant Cell. 2012;24:4731-47.

35. Town CD, Cheung F, Maiti R, Crabtree J, Haas BJ, Wortman JR, et al. Comparative genomics of Brassica oleracea and Arabidopsis thaliana reveal gene loss, fragmentation, and dispersal after polyploidy. Plant Cell. 2006;18: 1348-59.

36. Yang TJ, Kim JS, Kwon SJ, Lim KB, Choi BS, Kim JA, et al. Sequence-level analysis of the diploidization process in the triplicated FLOWERING LOCUS C region of Brassica rapa. Plant Cell. 2006;18:1339-47.

37. Blanc $\mathrm{G}$, Hokamp $\mathrm{K}$, Wolfe $\mathrm{KH}$. A recent polyploidy superimposed on older large-scale duplications in the Arabidopsis genome. Genome Res. 2003;13: $137-44$.

38. Lysak MA, Koch MA, Pecinka A, Schubert I. Chromosome triplication found across the tribe Brassiceae. Genome Res. 2005;15:516-25.

39. Graham GJ. Tandem genes and clustered genes. J Theor Biol. 1995;175:7187.

40. Chalhoub B, Denoeud F, Liu SY, Parkin IAP, Tang HB, Wang XY, et al. Early allopolyploid evolution in the post-Neolithic Brassica napus oilseed genome. Science. 2014;345:950-3.

41. Liu SY, Liu YM, Yang XH, Tong CB, Edwards D, Parkin IAP, Zhao MX, Ma JX, Yu JY, Huang SM, et al. The Brassica oleracea genome reveals the asymmetrical evolution of polyploidy genomes. Nat Commun. 2014;5:3930.

42. Kaewthai N, Gendre D, EklöF JM, Ibatullin FM, Ezcuura I, Bhalerao RP, et al. Group III-A XTH genes of Arabidopsis encode predominant xyloglucan endohydrolases that are dispensable for normal growth. Plant Physiol. 2013; 161(1):440-54.

43. Yokoyama R, Nishitani K. Functional diversity of xyloglucan-related proteins and its implications in the cell wall dynamics in plants. Plant Biol. 2000;2: 598-604.

44. Kallas AM, et al. Enzymatic properties of native and deglycosylated hybrid aspen (Populus tremula $x$ tremuloides) xyloglucan endotransglycosylase 16A expressed in Pichia pastoris. Biochem J. 2005;390:105-13.

45. Johnasson P, Brumer H, Baumann MJ, Kallas ÅM, Henriksson H, Denman SE, et al. Crystal structures of a poplar Xyloglucan Endotransglycosylase reveal details of Transglycosylation acceptor binding. Plant Cell. 2004;16(4):874-86.

46. Henriksson H, Denman SE, Campuzano IDG, Ademark P, Master ER, Teeri TT, et al. N-linked glycosylation of native and recombinant cauliflower xyloglucan endotransglycosylase 16A. Biochem J. 2003;375(1):61-73.

47. Kozak KH, Mendyk RW, Wiens JJ. Can parallel diversification occur in sympatry? Repeated patterns of body-size evolution in coexisting clades of north American salamanders. Evolution. 2009;63(7):1769-84.

48. Cheng F, Wu J, Fang L, Wang X. Syntenic gene analysis between B rapa and other Brassicaceae species. Front Plant Sci. 2012;3:198.

49. Wang $X$, Wang $H$, Wang J, Sun $R$, Wu J, Liu S, et al. B. rapa genome sequencing project consortium-the genome of the mesopolyploid crop species Brassica rapa. Nat Genet. 2011:43:1035-9.

50. Nekrutenko A, Makova KD, Li WH. The KA/KS ratio test for assessing the protein-coding potential of genomic regions: an empirical and simulation study. Genome Res. 2002;12:198-202.

51. Rai KM, Thu SW, Balasubramanian VK, Cobos CJ, Disasa T, Mendu V. Identification, Characterization, and Expression Analysis of Cell Wall Related Genes in Sorghum bicolor (L.) Moench, a Food, Fodder, and Biofuel Crop. Front Plant Sci. 2016;7(77):1287.

52. Nawaz MA, Rehman HM, Imtiaz M, Baloch FS, Lee JD, Yang SH, et al. Systems Identification and Characterization of Cell Wall Reassembly and Degradation Related Genes in Glycine max (L.) Merill, a Bioenergy Legume. Sci Rep. 2017;7:10862.

53. Wolf $Y$, Madej T, Babenko V, Shoemaker B, Panchenko AR. Long-term trends in evolution of indels in protein sequences. BMC Evol Biol. 2007;7:19.

54. Van Sandt V, Guisez Y, Verbelen JP, Vissenberg K. Analysis of a xyloglucan endotransglucosylase/hydrolase (XTH) from the lycopodiophyte Selaginella kraussiana suggests that XTH sequence characteristics and function are 
highly conserved during the evolution of vascular plants. J Exp Bot. 2006;57: 2909-22.

55. Liang ZW, Li MD, Liu ZHY, Wang JB. Genome-wide identification and characterization of the Hsp70 gene family in allopolyploid rapeseed (Brassica napus L.) compared with its diploid progenitors. Peer J. 2019;7:e7511.

56. Becnel J, Natarajan M, Kipp A, Braam J. Developmental expression patterns of Arabidopsis XTH genes reported by transgenes and Genevestigator. Plant Mol Biol. 2006;61:451-67.

57. Lynch M, Conery JS. The evolutionary fate and consequences of duplicate genes. Science. 2000;290:1151-5.

58. Cheng F, Liu SY, Wu J, Fang L, Sun SL, Liu B, et al. BRAD, the genetics and genomics database for Brassica plants. BMC Plant Biol. 2011;11:136.

59. Lamesch P, Berardini TZ, Li D, Swarbreck D, Wilks C, Sasidharan R, et al. The Arabidopsis information resource (TAIR): improved gene annotation and new tools. Nucleic Acids Res. 2012:40:D1202-10

60. Finn RD, Coggill P, Eberhardt RY, Eddy SR, Mistry J, Mitchell AL, et al. The Pfam protein families database: towards a more sustainable future. Nucleic Acids Res. 2016;44:D279-85.

61. Chen CJ, Chen H, Zhang Y, Thomas HR, Frank MH, He YH, et al. TBtools - an integrative toolkit developed for interactive analyses of big biological data. Mol Plant. 2020. https://doi.org/10.1016/jmolp202006009.

62. Marchler-Bauer A, Bo Y, Han L, He J, Lanczycki CJ, Lu S, et al. CDD/SPARCLE: functional classification of proteins via subfamily domain architectures. Nucleic Acids Res. 2017;45:D200-3.

63. Østergaard L, King GJ. Standardized gene nomenclature for the Brassica genus. Plant Methods. 2008:4:10.

64. Wilkins MR, Gasteiger E, Bairoch A, Sanchez JC, Williams KL, Appel RD, et al. Protein identification and analysis tools in the ExPASy server. Methods Mol Biol. 1999;112:531-52.

65. Chou KC, Shen HB. Cell-PLoc 2.0: An improved package of web-servers for predicting subcellular localization of proteins in various organisms. Nat Sci. 2010;2: 1090-103.

66. Armenteros JJA, Tsirigos KD, Sonderby CK, Petersen TN, Winther O, Brunak S, et al. SignalP 5.0 improves signal peptide predictions using deep neural networks. Nat Biotechnol. 2019:37:420.

67. Bailey TL, Johnson J, Grant CE, Noble WS. The MEME suite. Nucleic Acids Res. 2015:43:W39-49.

68. Kumar S, Stecher G, Tamura K. MEGA7: molecular evolutionary genetics analysis version 7.0 for bigger datasets. Mol Biol Evol. 2016;33:1870-4.

69. Letunic I, Bork P. Interactive tree of life (ITOL) V3: an online tool for the display and annotation of phylogenetic and other trees. Nucleic Acids Res. 2016;44:W242-5.

70. Robert $X$, Gouet P. Deciphering key features in protein structures with the new ENDscript server. Nucleic Acids Res. 2014;42(W1):W320-4.

71. Voorrips RE. Mapchart: software for the graphical presentation of linkage maps and QTLs. J Hered. 2002;93:77-8.

72. Kong X, Lv W, Jiang S, Zhang D, Cai G, Pan J, et al. Genome-wide identification and expression analysis of calcium-dependent protein kinase in maize. BMC Genomics. 2013;14:433.

73. Yu J, Ke T, Tehrim S, Sun F, Liao B, Hua W. PTGBase: an integrated database to study tandem duplicated genes in plants. Database (Oxford). 2015;2015: bav017. https://doi.org/10.1093/database/bav017.

74. Doerks T, Copley RR, Schultz J, Ponting CP, Bork P. Systematic identification of novel protein domain families associated with nuclear functions. Genome Res. 2002;12:47-56.

75. Koch MA, Haubold B, Mitchell-Olds T. Comparative evolutionary analysis of chalcone synthase and alcohol dehydrogenase loci in Arabidopsis, Arabis, and related genera (Brassicaceae). Mol Biol Evol. 2000;17:1483-98.

76. Yu J, Tehrim S, Zhang F, Tong C, Huang J, Cheng X, et al. Genome-wide comparative analysis of NBS-encoding genes between Brassica species and Arabidopsis thaliana. BMC Genomics. 2014;15(1):3.

77. Tong C, Wang X, Yu J, Wu J, Li W, Huang J, et al. Comprehensive analysis of RNA-seq data reveals the complexity of the transcriptome in Brassica rapa. BMC Genomics. 2013;14:689.

78. Yao QY, Xia EH, Liu FH, Gao LZ. Genome-wide identification and comparative expression analysis reveal a rapid expansion and functional divergence of duplicated genes in the WRKY gene family of cabbage, Brassica oleracea var capitata. Gene. 2015:35:557.

\section{Publisher's Note}

Springer Nature remains neutral with regard to jurisdictional claims in published maps and institutional affiliations.

Ready to submit your research? Choose BMC and benefit from:

- fast, convenient online submission

- thorough peer review by experienced researchers in your field

- rapid publication on acceptance

- support for research data, including large and complex data types

- gold Open Access which fosters wider collaboration and increased citations

- maximum visibility for your research: over $100 \mathrm{M}$ website views per year

At BMC, research is always in progress.

Learn more biomedcentral.com/submissions 